\title{
OPEN FDG PET biomarkers for prediction of survival in metastatic melanoma prior to anti-PD1 immunotherapy
}

\author{
A. Flaus $\oplus^{1,2,8 \bowtie}$, V. Habouzit ${ }^{1}$, N. De Leiris ${ }^{3,4}$, J. P. Vuillez ${ }^{3,4}$, M. T. Leccia ${ }^{5}$, J. L. Perrot ${ }^{6}$, \\ N. Prevot $^{1} \&$ F. Cachin ${ }^{7}$
}

Our aim was to analyse whether biomarkers extracted from baseline ${ }^{18}$ F-FDG PET before anti-PD1 treatment contribute to prognostic survival information for early risk stratification in metastatic melanoma. Fifty-six patients, without prior systemic treatment, BRAF wild type, explored using ${ }^{18} \mathrm{~F}$-FDG PET were included retrospectively. Our primary endpoint was overall survival (OS). Total metabolic tumoral volume (MTV) and forty-one IBSI compliant parameters were extracted from PET. Parameters associated with outcome were evaluated by a cox regression model and when significant helped build a prognostic score. Median follow-up was 22.1 months and 21 patients died. Total MTV and long zone emphasis (LZE) correlated with shorter OS and served to define three risk categories for the prognostic score. For low, intermediate and high risk groups, survival rates were respectively $91.1 \%$ (IC 95 80-1), 56.1\% (IC 95 37.1-85) and 19\% (IC $950.06-60.2$ ) and hazard ratios were respectively 0.11 (IC $950.025-0.46$ ), $P=0.0028,1.2$ (IC $950.48-2.8$ ), $P=0.74$ and 5.9 (IC 95 2.5-14), $P<0.0001$. To conclude, a prognostic score based on total MTV and LZE separated metastatic melanoma patients in 3 categories with dramatically different outcomes. Innovative therapies should be tested in the group with the lowest prognosis score for future clinical trials.

Immunotherapy using immune checkpoint inhibitors (ICI) targeting programmed cell death 1 receptor (PD-1) is now a well-established treatment for patients with metastatic melanoma ${ }^{1}$. Indeed, clinical benefits and durability of treatment response outperform standard chemotherapy ${ }^{2}$.

However, only subsets of patients will benefit from it ${ }^{1}$ : thus, we need to develop tools predicting patient survival after immunotherapy to prevent toxicities and hasten the introduction of more appropriate treatments when necessary. To answer this question different biomarkers have been analysed such as levels of programmed cell death ligand 1 (PDL1) expression ${ }^{3}$, presence of tumour infiltrating lymphocytes ${ }^{4}$, genetic mutations ${ }^{5}$ and inflammatory cytokines ${ }^{6}$. Currently none are clinically identified as helpful for patient selection ${ }^{7}$.

Adopting the quantitative imaging biomarkers approach is an interesting alternative ${ }^{8}$. Indeed, various quantitative features can be extracted from images such as pixel intensity, shape and texture 9 .

${ }^{18}$ F-labeled fluorodeoxyglucose positron emission tomography -computed tomography $\left({ }^{18} \mathrm{~F}\right.$ FDG PET-CT) provides valuable functional information based on the increased glucose uptake and glycolysis of metastatic melanoma cells ${ }^{10} .{ }^{18} \mathrm{~F}$ FDG PET-CT is commonly used for the initial staging of metastatic melanomas.

For metastatic melanoma, previous studies in heterogenous cohorts of patients-treated either with anticytotoxic T lymphocytes associated protein (anti-CTLA 4$)^{11}$ or anti-PD1 $1^{12}$-have shown that total metabolic tumour volume (total MTV) from baseline ${ }^{18} \mathrm{~F}$ FDG PET-CT correlate with overall survival (OS). Similar results were found for non-small cell lung cancer patients treated by immunotherapy ${ }^{13}$. Moreover, textural feature from baseline ${ }^{18} \mathrm{~F}$ FDG PET-CT, such as tumour heterogeneity, were also linked with $\mathrm{OS}^{14}$. However, in this study total MTV was not computed, and authors included patients with various stages and different immunotherapies (anti-PD1 and anti-CTLA 4).

\footnotetext{
${ }^{1}$ Nuclear Medecine Department, Saint-Etienne University Hospital, University of Saint-Etienne, Saint-Etienne, France. ${ }^{2}$ Nuclear Medicine Department, East Group Hospital, Hospices Civils de Lyon, Lyon, France. ${ }^{3}$ Nuclear Medecine Department, CHU Grenoble Alpes, University Grenoble Alpes, Grenoble, France. ${ }^{4}$ Laboratoire Radiopharmaceutiques Biocliniques, University Grenoble Alpes, INSERM, CHU Grenoble Alpes, 38000 Grenoble, France. ${ }^{5}$ Dermatology Department, CHU Grenoble Alpes, University Grenoble Alpes, Grenoble, France. ${ }^{6}$ Dermatology Department, Saint-Etienne University Hospital, University of Saint-Etienne, Saint-Etienne, France. ${ }^{7}$ Nuclear Medicine Department, Jean Perrin Cancer Center of Clermont-Ferrand, Clermont-Ferrand, France. ${ }^{8}$ Service de Medecine Nucléaire, Hôpital Nord, CHU de Saint-Etienne, 42 055 Saint-Etienne, Cedex 2, France. ${ }^{\varpi}$ email: anthime.flaus@gmail.com
} 


\begin{tabular}{|l|l|l|l|l|l|}
\hline Scanner & Reconstruction & Iteration and subsets & Gaussian post-filtering & Matrix & Voxel size (x,y,z mm) \\
\hline $\begin{array}{l}\text { Siemens, Biograph Horizon } \\
16\end{array}$ & OSEM & 3 iterations and 10 subsets & FWHM 4 mm & 360 & $2.06 \times 2.06 \times 2.03$ \\
\hline GE, Discovery 690 & OSEM & 2 iterations and 24 subsets & FWHM 4 mm & 256 & $2.73 \times 2.73 \times 3.27$ \\
\hline Siemens, Biograph 20 mCT & OSEM & 2 iterations and 21 subsets & FWHM 5 mm & 256 & $1.59 \times 1.59 \times 3$ \\
\hline $\begin{array}{l}\text { Siemens, Biograph 6 } \\
\text { HI-REZ }\end{array}$ & OSEM & 4 iterations and 8 subsets & FWHM 5 mm & 256 & $4.06 \times 4.06 \times 4$ \\
\hline
\end{tabular}

Table 1. Image reconstruction parameters for all four different PET systems. OSEM ordered subsets expectation maximization, FWHM full width at half maximum.

The aim of our work is to analyse whether biomarkers-extracted from baseline ${ }^{18} \mathrm{~F}$ FDG PET-CT performed prior to anti-PD1 treatment-can improve information on survival and prognosis in a highly selected cohort of patients with metastatic melanoma.

\section{Materials and methods}

Source of data. The hospital information system of two university hospitals (University hospital of SaintEtienne and Grenoble) was questioned to identify metastatic melanoma patients treated with first-line anti-PD1 treatment and imaged with a ${ }^{18}$ F-FDG PET-CT scan before therapy between January 2016 and January 2019. Electronic clinical and radiological databases were used to obtain patient demographic details, clinical history, anatomopathological data, treatment data, clinical outcome and follow-up duration as well as ${ }^{18} \mathrm{~F}$-FDG PET data.

The protocol was approved by the institutional medical ethics committee (IRB: IORG0007394) of SaintEtienne (IRBN 842,020/CHUSTE) and all methods were carried out in accordance with relevant guidelines and regulations. Informed consent was obtained from all the patients to participate in the study according to national regulations.

Patients. Out of sixty-four screened patients, fifty-six were included. Inclusion criteria were as follows: (1) biopsy-proven metastatic melanoma; (2) anti-PD1 antibodies were the first line of treatment, with no previous systematic therapy; (3) patients were B-RAF wild type; (4) pre-treatment ${ }^{18} \mathrm{~F}$-FDG PET data were available. Eight patients were excluded: patients with no measurable disease or no significant FDG avid tumour $(n=6)$. It included patients presenting only brain metastases due to the impossibility of clear delineation of metabolic tumoral volume, and patients with lesion sizes inferior to 64 voxels $(n=2)$, the radiomic analysis becoming irrelevant below this threshold ${ }^{15,16}$.

Metastatic status was classified as defined by the eighth edition American Joint Committee on Cancer (AJCC) melanoma staging system ${ }^{17}$ :

- M1a: distant metastasis to skin, soft tissue including muscles, and/or nonregional lymph

- M1b: distant metastasis to lung with or without M1a sites of disease

- M1c: distant metastasis to non-central nervous system (CNS) visceral sites with or without M1a or M1b sites of disease

- M1d: distant metastasis to CNS with or without M1a, M1b, or M1c sites of disease.

Outcome: overall survival. OS was defined as the time (in months) between the ${ }^{18} \mathrm{~F}-\mathrm{FDG}$ PET scan and the date of death of any cause. The length of the follow up was calculated starting from the date of the initial ${ }^{18} \mathrm{~F}-\mathrm{FDG}$ PET up to the date of the last clinical consultation. Living patients were censored at the time of the last clinical follow-up.

${ }^{18}$ F-FDG PET-CT protocol. Scans were acquired with different PET-CT systems: Biograph mCT Flow 20 (Siemens Healthcare) and Biograph 6 HI-REZ (Siemens Healthcare) were used at the first University Hospital. Biograph Horizon 16 (Siemens Healthcare) and Discovery 690 (General Electrics Healthcare,) were employed at the second University Hospital.

Patients fasted for at least $6 \mathrm{~h}$ and blood glucose was $<180 \mathrm{mg} / \mathrm{dl}$. Patients were injected according to current guidelines with an activity of $2.5-4 \mathrm{MBq} / \mathrm{kg}$ of ${ }^{18} \mathrm{~F}-\mathrm{FDG}$ (median activity $269 \mathrm{MBq}$, range 146-468). Sixty minutes after injection whole-body PET and unenhanced CT images were acquired. Images were reconstructed using iterative algorithms and using CT for attenuation correction. As shown in Table 1, image reconstruction parameters were different but consistent for each PET scanner. Using the following formula, each voxel in PET images was converted to standard uptake value (SUV) : SUV = voxel concentration activity ${ }^{*}$ patient body weight / decay corrected injected activity ${ }^{18}$.

Measuring imaging biomarkers. We performed segmentation and the quantitative features' extraction process using the free software LIFEx (v4.0, Local Image Feature Extraction) which complies with the image biomarker standardization initiative (IBSI) ${ }^{16,19}$. One experienced nuclear medicine physician analysed and segmented ${ }^{18}$ F-FDG PET scans without knowledge of the patients' clinical outcome. Based on a $40 \%$ SUVmax threshold, a semi-automatic method was employed for segmentation ${ }^{20}$. 
Total metabolic tumour volume. Each hypermetabolic lesion was segmented to create a volume of interest (VOI) corresponding to the MTV. SUVmean was measured as the average uptake in the tumour VOI. A total MTV per patient was defined as the sum of the MTV of each hypermetabolic metastatic lesion ${ }^{11,12}$.

Conventional and Textural PET parameters. For each patient, quantitative features were extracted from the VOI of the lesion with the highest ${ }^{18} \mathrm{~F}$-FDG uptake. Before textural feature calculation, voxel intensities of the VOI were resampled using 64 discrete values ranging from 0 to $32 \mathrm{SUV}$ units ${ }^{21} .41$ features in total were extracted: five conventional PET parameters-SUVmax, SUVmean, SUVmin, SUVpeak and Total lesion glycolysis (TLG) - five descriptors of the image intensity histogram, and $31 \mathrm{~s}$ order textural features-six from the Grey Level CoOccurrence matrix (GLCM), eleven from the Grey-level run length matrix (GLRLM), three from the Neighbourhood grey-level different matrix (NGLDM) and eleven from the Grey-level zone length matrix (GLZLM). A detailed description of each parameter is available in the technical appendix of the LIFEx software ${ }^{16}$.

Feature pre-processing. As the study was retrospective no pre acquisition harmonisation was performed. However, in order to pool together conventional and textural features from the four different PET/CT scanners, ComBat post-reconstruction harmonization method was used ${ }^{22,23}$. After ComBat harmonization, a feature selection was used to shrink the model, reducing overfitting and co-variate correlation. Features' bilateral correlation were evaluated with Spearman's rank correlation coefficient ${ }^{24}$ and those with correlation coefficient higher than 0.8 were removed ${ }^{25}$.

Statistical analysis. For statistical analyses and plotting the open-source $R$ software package was utilized ${ }^{26}$ (version 3.0.1, http://www.Rproject.org). Continuous variables are presented as median [range] and were compared using Wilcoxon rank sum test. Discrete variables are presented as number and percentage and were compared using chi-test or fisher-test if the amount of data were below 5 .

In order to select PET parameters for further analysis, we compared mean values of pre-treatment PET parameters according to OS status at the time of analysis using two sided Wilcoxon-Mann Whitney tests because these parameters did not follow a Gaussian distribution $(P<0.0035$ was considered as statistically significant after Bonferroni correction). Only those that were statistically significant were selected for further analysis and optimal cut-off for each significant parameters was based on the receiver operating characteristic (ROC) curves (pROC ${ }^{27}$ package, version 1.17.0.1, http://expasy.org/tools/pROC/).

Selected PET biomarkers, age, gender, and metastatic status were included in univariate and multivariate Cox regression models. Because of the small number of events, the multivariable analysis was restricted to variables that displayed a univariate analysis' Hazard Ratio (HR) with a p value below 0.05 . Kaplan Meier curves for OS were built for variables found significant during the multivariate analysis and compared with log-rank tests $(P<0.05$ was considered statistically significant). Survival rates were calculated for each group at the end of the follow-up period (Survival package, version 3.2-7, https://CRAN.R-project.org/package=survival).

With the aim of stratifying the population into different risk categories, we constructed a prognostic composite metabolic score based on the parameters we found significant in the Cox models. Kaplan Meier analyses were computed for these different risk groups and compared with log-rank test $(P<0.05$ was considered statistically significant).

\section{Results}

Patient characteristics. Pre-treatment patient characteristics are summarized in Table 2. Fifty-six patients with wild-type BRAF metastatic melanoma were included with a median age of 68 years old (range: 40-84). They all received first-line anti-PD1 therapies (Nivolumab or Pembrolizumab) as their first systemic treatment. At the time of analysis, the median follow-up was 22.1 months (range: $2.1-49.2$ ) and 21 patients (38\%) had died. There were no statistically significant differences between groups of patients alive and dead at time of analysis about sex $(P=0.3)$, age $(P=0.99)$, university hospital $(P=0.94)$, metastatic status before the start of anti-PD1 treatment $(P=0.91)$, anatomopathological characteristic of initial melanoma lesion such as Breslow $(P=0.56)$, presence of ulceration $(P=0.81)$, high rate of mitosis $\left(>1 / \mathrm{mm}^{2}\right)(P=0.97)$, localisation $(P=0.88)$ and the initial cancer staging $(P=0.57)$ (Supplementary Table 2$)$.

PET biomarkers correlated with survival. We correlated ${ }^{18} \mathrm{~F}-\mathrm{FDG}$ PET biomarkers with OS status in patients treated with anti-PD1. Only mean total MTV and mean Long Zone Emphasis (LZE) differed significantly between patients that were alive or dead at the time of the analysis (Supplementary Table 1). Mean total MTV burden was significantly lower in the group of patients alive at the time of analysis (mean $18.22 \mathrm{~cm}^{3}$, IC95 7.08-29.36 vs. $49.69 \mathrm{~cm}^{3}$, IC95 $16.88-82.49 ; P=0.001$ ). To identify patients with the worse prognosis, the optimal mean total MTV cut-off value was $5.6 \mathrm{~cm}^{3}$, with a sensitivity of 0.9 and a specificity of 0.63 (ROC curve $\mathrm{AUC}=0.76$, CI95 0.64-0.89; Supplementary Fig. 1a). Figure 1 represents maximum intensity projection PET images from two subjects. (A) alive at the time of analysis with low total MTV $\left(3 \mathrm{~cm}^{3}\right)$ and low LZE $(-4194)$. (B) dead at the time of analysis with high total MTV $(50,5)$ and high LZE $(58,336)$. Moreover, mean LZE, extracted from the GLZLM matrix, was significantly lower in the group of patients alive at the time of analysis $(-1040.60$, CI95 - 5615.21-3535 vs. 10,507.6, CI95 2309.75-18,705.44, $P=0.003$ ). The optimal mean LZE cut-off value to identify worse prognostic patients was-437, with a sensitivity of 0.62 and a specificity of 0.89 (ROC curve AUC=0.73, CI95 0.57-0.89; Supplementary Fig. 1b).

In univariate analysis, only high total MTV $(P=0.002)$ and high LZE $(P<0.001)$ were significantly correlated with poor OS. No clinical parameters were significantly associated with the outcome (Table 3 ). Looking at the multivariate analysis, high total MTV and high LZE remained statistically significant independent prognostic 


\begin{tabular}{|c|c|}
\hline Characteristics & Median[range], n(\%) \\
\hline \multicolumn{2}{|l|}{ Clinical characteristics } \\
\hline \multicolumn{2}{|l|}{ Demographic parameters } \\
\hline Age (years) & $68[40-84]$ \\
\hline Female & $27(48 \%)$ \\
\hline Male & $29(52 \%)$ \\
\hline \multicolumn{2}{|l|}{ PET Centre } \\
\hline Saint-Etienne PET Centre & $33(59 \%)$ \\
\hline Siemens, Biograph 6 HI-REZ & $14(25 \%)$ \\
\hline Siemens, Biograph $20 \mathrm{mCT}$ & $19(34 \%)$ \\
\hline Grenoble PET Centre & $23(41 \%)$ \\
\hline GE, Discovery 690 & $12(21 \%)$ \\
\hline Siemens, Biograph Horizon 16 & $11(20 \%)$ \\
\hline \multicolumn{2}{|l|}{ Metastatic status (8th AJCC classification) } \\
\hline M1a & $13(23 \%)$ \\
\hline M1b & $5(9 \%)$ \\
\hline M1c & $31(55 \%)$ \\
\hline M1d & $7(13 \%)$ \\
\hline \multicolumn{2}{|l|}{ Treatment } \\
\hline Median duration between PET and start of treatment (months) & $1.1[0.03-9.07]$ \\
\hline \multicolumn{2}{|l|}{ Follow-up } \\
\hline Duration (months) & $22.1[2.1-49.2]$ \\
\hline \multicolumn{2}{|l|}{ Survival } \\
\hline Death & $21(38 \%)$ \\
\hline
\end{tabular}

Table 2. Clinical characteristics of the patients $(n=56)$. SUV standardized uptake value, PET positron emission tomography, $M$ Metastatic status (8th American Joint Committee on Cancer classification): M1a: distant metastasis to skin, soft tissue including muscles, and/or nonregional lymph. M1b: distant metastasis to lung with or without M1a sites of disease. M1c: distant metastasis to non-central nervous system (CNS) visceral sites with or without M1a or M1b sites of disease. M1d: distant metastasis to CNS with or without M1a, M1b, or M1c sites of disease.

factors for OS (HR 4.1 CI95 1.1-15.3, $P=0.034$ and HR 3.7 CI95 1.5-9.5, $P=0.006$, respectively) (Table 3). Using log rank test, Kaplan Meier curves comparing overall survival in patients with high versus low total MTV and high versus low LZE were significantly different, with respective p values of 0.00038 and $<0.0001$ (Supplementary Fig. 2). Survival rates were $87.6 \%$ (CI95 75.4-1) for low MTV versus 40.3\% for high MTV (CI 95 25.9-62.8). Survival rates were 77\% (CI 95 64.8-91.4) for low LZE versus 55.2\% for high LZE (CI 95 7.1-61.4).

Prognostic composite metabolic score. Since total MTV and LZE are both independent prognostic factors for OS in our multivariate analysis, we developed a prognostic score by combining them. The population was therefore stratified in three risk categories: low risk if total MTV $\leq 5.6 \mathrm{~cm}^{3}$ and LZE $\leq-437(\mathrm{n}=23 ; 41 \%)$, intermediate risk if total MTV $>5.6 \mathrm{~cm}^{3}$ or LZE $>-437(\mathrm{n}=19,34 \%)$ and high risk if total MTV $>5.6 \mathrm{~cm}^{3}$ and LZE $>-437(n=14 ; 25 \%)$.

Survival rates were respectively $91.1 \%$ (CI 95 80-1) for the low risk group, $56.1 \%$ for the intermediate risk group (CI 95 37.1-85) and 19\% for the high risk group (CI 95 0.06-60.2). HR for OS were respectively 0.11 for the low risk group (CI95 0.025-0.46), $P=0.0028,1.2$ for the intermediate risk group (CI $950.48-2.8$ ), $P=0.74$ and 5.9 for the high risk group (CI $952.5-14$ ), $P<0.0001$.

Figure 2 represents Kaplan Meier curves of OS according to the three risk categories $(P<0.0001)$. Log rank tests differed significantly between Kaplan Meier curves for OS of low versus intermediate-risk groups $(P=0.008)$, low versus high-risk groups $(P<0.001)$, and intermediate versus high-risk groups $(P=0.013)$ (Supplementary Fig. 3).

\section{Discussion}

Our results add to the accumulating evidence that beyond initial staging, ${ }^{18} \mathrm{~F}-\mathrm{FDG}$ PET-CT scan provides simple to use and cost-effective biomarkers associated with OS. LZE and total MTV demonstrated their high prognostic value in metastatic melanoma patients treated with anti-PD1. As the former was highly specific, the latter highly sensitive, and they were not correlated, we used both parameters to build three risk categories, creating a prognostic composite metabolic score. For low, intermediate and high-risk groups, survival rates were respectively $91.1 \%$ (CI 95 80-1), 56.1\% (CI 95 37.1-85) and 19\% (CI 95 0.06-60.2). These imaging metrics provide a novel method for improving the stratification and selection of patients who would most benefit from additional immunotherapies beyond anti-PD1. 

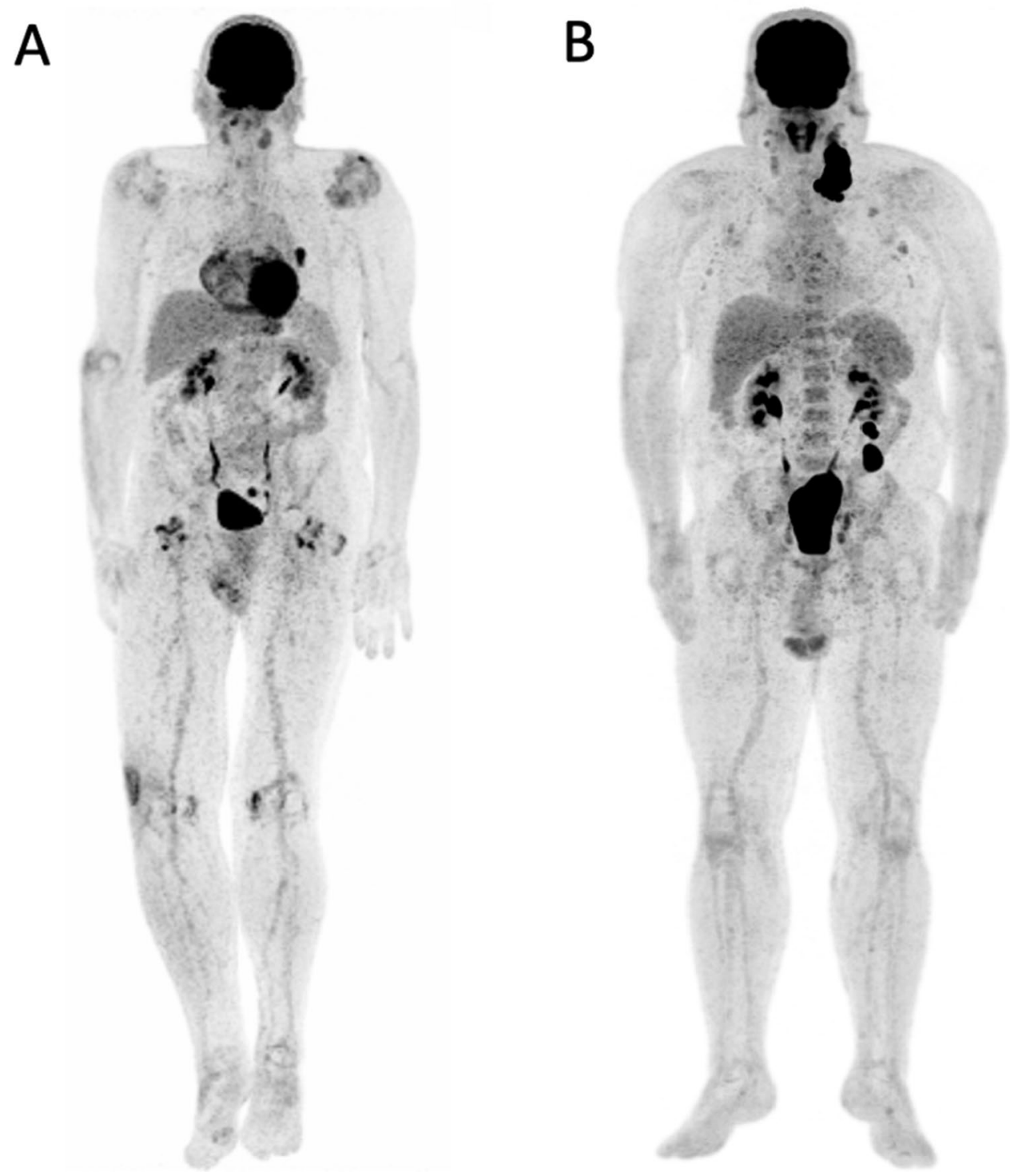

Figure 1. The maximum intensity projection PET images from two subjects. (A) Alive at the time of analysis, showed an inguinal right lymph node and a left pulmonary nodule (total MTV $3 \mathrm{~cm}^{3}$ ) with low LZE (-4194) and (B) dead at the time of analysis, showed cervical, axillary, celiac, mesenteric, iliac lymph nodes (total MTV $\left.50,5 \mathrm{~cm}^{3}\right)$ with high LZE $(58,336)$. Total MTV: total metabolic tumoral volume, LZE: long zone emphasis.

In our study, patients with a high metabolic tumour burden (total MTV $>5.6 \mathrm{~cm}^{3}$ ) exhibited a poorer prognostic, with a shorter median OS, strengthening the prognostic significance of pre-treatment total MTV before anti-PD1 therapies ${ }^{28}$. Previous studies found similar results in heterogeneous cohorts of melanoma patients, irrespectively of their BRAF status. In the first study, 55 patients underwent ${ }^{18} \mathrm{~F}$-FDG PET before anti-PD1 as a first or further line treatment : high total MTV on baseline imaging was significantly correlated with a shorter survival (in multivariable analysis) with a cut-off value set at $25 \mathrm{~cm}^{312}$. In a second retrospective cohort, 142 patients underwent ${ }^{18}$ F-FDG PET before anti-CTL4 as a first line or further line treatment : high total MTV was significantly correlated with OS (after multivariate analysis), with a cut-off value set at $26.85 \mathrm{~cm}^{311}$. In our study, the total MTV cut-off was smaller because our population was limited to patients who received anti-PD1 as a first line therapy. To the best of our knowledge, our study is the first to analyse the prognostic value of total MTV in this specific sub-group of patients with metastatic melanoma, thus extending its validity as a prognostic biomarker.

Textural feature, LZE, brought additional prognostic information as it allowed a reclassification of patients into an intermediate risk group (total MTV $>5.6 \mathrm{~cm}^{3}$ or LZE $>-437$ ) which encompassed $34 \%$ of the population $(n=19)$. Seventeen of these nineteen patients exhibited a high total MTV. No correlation was found between total MTV and LZE values, suggesting that LZE status dichotomize high total MTV patients in two sub-groups with a different prognosis.

LZE measures the distribution of long homogeneous grey level zones in the image irrespectively of their intensity: therefore, LZE reflects the lesion's heterogeneity. In our study, patients with high value of tumoral LZE 


\begin{tabular}{|l|l|l|l|l|l|l|}
\hline \multirow{2}{*}{ Variable } & \multicolumn{4}{l}{ Univariate analysis } & \multicolumn{4}{l|}{ Multivariate analysis } \\
\cline { 2 - 7 } & HR & $\mathbf{9 5 \%} \mathbf{C I}$ & $\boldsymbol{p}$-Value & HR & $\mathbf{9 5 \%}$ CI & $\boldsymbol{P}$-value \\
\hline Age & 1 & $1-1$ & 0.96 & - & - & - \\
\hline Sex & 0.65 & $0.3-1.6$ & 0.33 & - & - & - \\
\hline M status & & & & - & - & - \\
\hline M1a vs. M1b & 0.4 & $0.05-3.4$ & 0.8 & & & \\
\hline M1a vs. M1c & 0.85 & $0.3-2.4$ & 0.9 & & & \\
\hline M1a vs. M1d & 0.97 & $0.2-4$ & 0.9 & & & \\
\hline LZE (GLZLM) & 6.4 & $2.6-16$ & $<0.001$ & 3.7 & $1.5-9.5$ & 0.006 \\
\hline Total MTV & 6.3 & $1,9-21$ & 0.003 & 4.1 & $1.1-15.3$ & 0.034 \\
\hline
\end{tabular}

Table 3. Univariate and multivariate cox regression analyses of overall survival in patients with metastatic melanoma. The distribution of each ${ }^{18} \mathrm{~F}$-FDG PET biomarker is a continuous variable that is transformed into a discrete categorization of 2 categories (high vs. low) using the values derived from the Youden's index: LZE $(\geq-437$ vs $<-437)$, total MTV ( $\geq 5.6$ vs $\left.<5.6 \mathrm{~cm}^{3}\right)$. HR hazard ratio, CI confidence interval, LZE (GLZLM) Long zone emphasis from the grey-level zone length matrix long-zone, $M T V$ metabolic tumoral volume, NGLDM Neighbourhood grey-level different matrix, TLG total lesion glycolysis, $M$ Metastatic status (8th American Joint Committee on Cancer classification): M1a: distant metastasis to skin, soft tissue including muscles, and/or nonregional lymph. M1b: distant metastasis to lung with or without M1a sites of disease. M1c: distant metastasis to non-central nervous system (CNS) visceral sites with or without M1a or M1b sites of disease. M1d: distant metastasis to CNS with or without M1a, M1b, or M1c sites of disease.

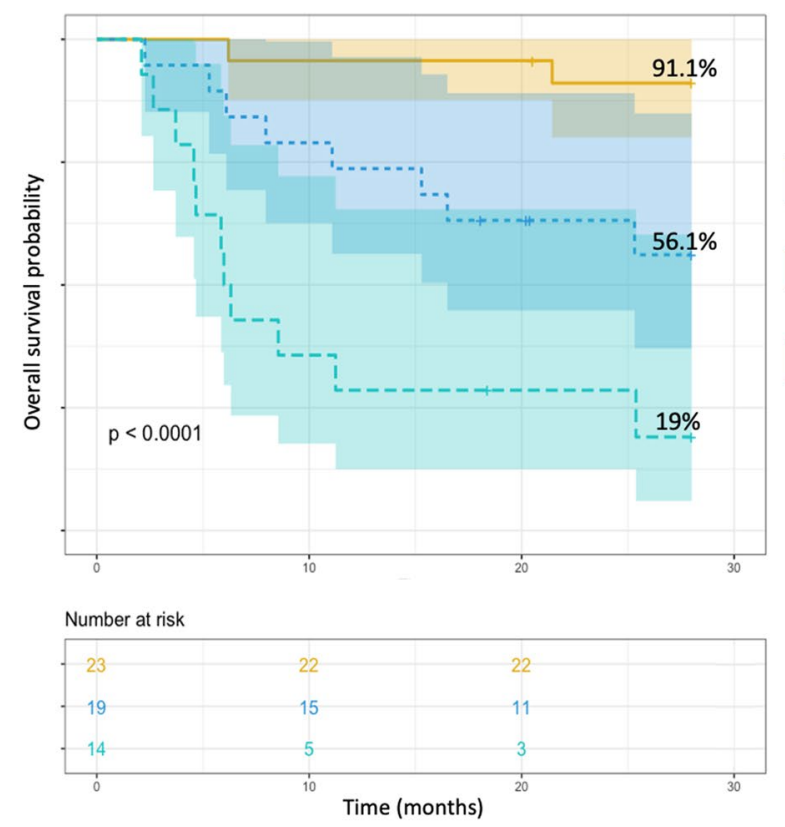

Figure 2. Kaplan Meier curves of overall survival (OS) based on a metabolic score combining total metabolic tumoral volume (total MTV) and long zone emphasis (LZE). The combined score comprising two binary risk variables was defined as follows: 0 risk variable = low risk group (total MTV $\leq 5.6 \mathrm{~cm}^{3}$ and LZE $\leq-437$ ), 1 risk variable $=$ intermediate risk group (total MTV $>5.6 \mathrm{~cm}^{3}$ or LZE $\left.>-437\right)$ and 2 risk variables $=$ high risk group (total MTV $>5.6 \mathrm{~cm}^{3}$ and LZE $>-437$ ).

showed poorer survival. Despite this promising finding, it is difficult to interpret the underlying mechanism translated by this heterogeneity parameter, which is based on a mathematical equation. Further investigations are required to understand the biological mechanisms underlying these heterogeneity parameters.

A metabolic score combining total MTV and LZE enhanced the prognostic value of ${ }^{18} \mathrm{~F}$-FDG PET imaging. Three different prognostic groups were identified. Low risk patients represented $41 \%$ of the population. Survival rate was $91.1 \%$ (CI 95 80-1) suggesting it is a biomarker for favourable outcome for anti-PD1 treatment. On the contrary, high-risk patients (25\%) had a poorer prognosis since their survival rate was 19\% (CI 95 0.06-60.2). So, our composite score appears as a biomarker, identifying patients who will not benefit from anti-PD1 therapy. This promising and innovative score provides the proof of concept for treatment personalization and should be validated in a larger, independent and prospective cohort. 
Previous studies suggested that textural parameters from ${ }^{18} \mathrm{~F}$-FDG PET-CT could predict outcome in various cancer types ${ }^{29-32}$ as it might represent tumour microenvironment, a known factor correlating with patients' response to anti-PD1 and anti-PDL1 ${ }^{33-35}$. Few studies in heterogeneous cohorts have investigated the potential for radiomics-extracted from ${ }^{18} \mathrm{~F}$-FDG PET images-to predict outcomes in metastatic melanoma after immunotherapy, and their results are contradictory ${ }^{12,14,36}$. For one study, the predictive value of SUV heterogeneity did not show prognostic value in an heterogeneous cohort of 55 melanoma patients treated by anti-PD ${ }^{12}$. However, in other studies textural parameters do carry prognostic information: in 34 patients with melanoma (stage from I to IV) treated with various immunotherapies (anti-PD1 and anti-CTLA4) as first or other line of treatment, gradient based tumour heterogeneity on pre-treatment ${ }^{18} \mathrm{~F}$-FDG PET correlated to survival outcome, as well as SUVmax and TLG. However as it was not compliant with the IBSI, comparison was limited ${ }^{14}$. Moreover, another study found a positive association between second order features and treatment response in 17 advanced melanoma patients treated respectively with anti-CTLA4 or Vemurafenib (PLX4032) according to their BRAF V600E status $^{36}$. In line with this study, we correlated LZE, a second order feature, with overall survival in patient with metastatic melanoma that were naïve of any previous systemic therapy prior to anti PD1. These data suggest that textural features, which are easily computed by free software, should be systematically assessed in prospective studies including ${ }^{18} \mathrm{~F}$ FDG PET-CT.

Our study has several limitations. Firstly, its design was retrospective and relatively few patients were included. Secondly, textural features exhibit varying sensitivity to the acquisition and reconstruction parameters and have previously limited multi PET scanner studies ${ }^{37}$. However in our work, the validated Combat harmonisation method was used ${ }^{22,23}$ to correct the effect of multi PET scanner as in other recent studies ${ }^{30,38}$. This method has proved its robustness even for a small number of patients per batch ${ }^{39}$. Thirdly the cut-offs that we used to create the 3 different risk groups are probably relying on PET acquisition system and reconstruction parameters. So it would be necessary to validate their robustness in a multicentric and prospective study. Lastly, as the study cohort was relatively small compared to the numbers of extracted features, Type 1 error rate may have increased. To prevent this, parameters highly inter-correlated were removed, thus decreasing redundant information, and we kept only independent parameters $(n=14$ vs. $n=42)$.

To conclude, we built a highly prognostic composite metabolic score based on LZE and total MTV values from baseline ${ }^{18}$ F FDG PET and used it to predict overall survival prior anti-PD1 therapy in a highly selected cohort of metastatic melanoma patients with no previous systemic treatment. This model could be implemented in clinical trials and then in clinical practice to stratify patients before treatment, because it represents a valuable biomarker that identifies patients who will not benefit from anti-PD1 therapy and therefore should switch to other treatment modalities.

\section{Data availability}

The datasets used and/or analysed during the current study are available from the corresponding author upon reasonable request.

Received: 17 May 2021; Accepted: 31 August 2021

Published online: 22 September 2021

\section{References}

1. Robert, C. et al. Nivolumab in previously untreated melanoma without BRAF mutation. N. Engl. J. Med. 372, 320-330 (2015).

2. Ribas, A. et al. Association of pembrolizumab with tumor response and survival among patients with advanced melanoma. JAMA 315, 1600-1609 (2016).

3. Meng, X., Huang, Z., Teng, F., Xing, L. \& Yu, J. Predictive biomarkers in PD-1/PD-L1 checkpoint blockade immunotherapy. Cancer Treat. Rev. 41, 868-876 (2015).

4. Zito Marino, F. et al. Are tumor-infiltrating lymphocytes protagonists or background actors in patient selection for cancer immunotherapy? Expert Opin. Biol. Ther. 17, 735-746 (2017).

5. Hellmann, M. D. et al. Nivolumab plus ipilimumab in lung cancer with a high tumor mutational burden. N. Engl. J. Med. 378, 2093-2104 (2018).

6. Ayers, M. et al. IFN- $\gamma$-related mRNA profile predicts clinical response to PD-1 blockade. J. Clin. Invest. 127, 2930-2940 (2017).

7. Hogan, S., Levesque, M. \& Cheng, P. Melanoma immunotherapy: next-generation biomarkers. Front. Oncol. 8, 178 (2018).

8. Durot, C. et al. Metastatic melanoma: pretreatment contrast-enhanced CT texture parameters as predictive biomarkers of survival in patients treated with pembrolizumab. Eur. Radiol. 29, 3183-3191 (2019).

9. Gillies, R. J., Kinahan, P. E. \& Hricak, H. Radiomics: images are more than pictures, they are data. Radiology 278, 563-577 (2016).

10. Ho, J. et al. Importance of glycolysis and oxidative phosphorylation in advanced melanoma. Mol. Cancer 11, 76 (2012).

11. Ito, K. et al. Prognostic value of baseline metabolic tumor volume measured on 18F-fluorodeoxyglucose positron emission tomography/computed tomography in melanoma patients treated with ipilimumab therapy. Eur. J. Nucl. Med. Mol. Imaging 46, 930-939 (2019).

12. Seban, R.-D. et al. Prognostic and theranostic 18F-FDG PET biomarkers for anti-PD1 immunotherapy in metastatic melanoma: association with outcome and transcriptomics. Eur. J. Nucl. Med. Mol. Imaging 46, 2298-2310 (2019).

13. Seban, R.-D. et al. FDG-PET biomarkers associated with long-term benefit from first-line immunotherapy in patients with advanced non-small cell lung cancer. Ann. Nucl. Med. 34, 968-974 (2020).

14. Sanli, Y., Leake, J., Odu, A., Xi, Y. \& Subramaniam, R. M. Tumor heterogeneity on FDG PET/CT and immunotherapy: an imaging biomarker for predicting treatment response in patients with metastatic melanoma. AJR Am. J. Roentgenol. 212, 1318-1326 (2019).

15. Hatt, M. et al. 18F-FDG PET uptake characterization through texture analysis: investigating the complementary nature of heterogeneity and functional tumor volume in a multi-cancer site patient cohort. J. Nucl. Med. 56, 38-44 (2015).

16. Nioche, C. et al. LIFEx: a freeware for radiomic feature calculation in multimodality imaging to accelerate advances in the characterization of tumor heterogeneity. Cancer Res. 78, 4786-4789 (2018).

17. Gershenwald JE, Scolyer RA, Hess KR, et al. Melanoma of the skin. In: Amin MB, Edge SB, Greene FL, et al., editors. AJCC Cancer Staging Manual. 8. 563-585 (Springer, 2017)

18. Thie, J. A. Understanding the standardized uptake value, its methods, and implications for usage. J. Nucl. Med. 45, 1431-1434 (2004). 
19. Zwanenburg, A. et al. The image biomarker standardization initiative: standardized quantitative radiomics for high-throughput image-based phenotyping. Radiology 295, 328-338 (2020).

20. Bashir, U. et al. The effects of segmentation algorithms on the measurement of (18)F-FDG PET texture parameters in non-small cell lung cancer. EJNMMI Res. 7, 60 (2017).

21. Orlhac, F., Soussan, M., Chouahnia, K., Martinod, E. \& Buvat, I. 18F-FDG PET-derived textural indices reflect tissue-specific uptake pattern in non-small cell lung cancer. PLoS ONE 10, e0145063 (2015).

22. Orlhac, F. et al. A postreconstruction harmonization method for multicenter radiomic studies in PET. J. Nucl. Med. 59, 1321-1328 (2018).

23. Mahon, R. N., Ghita, M., Hugo, G. D. \& Weiss, E. ComBat harmonization for radiomic features in independent phantom and lung cancer patient computed tomography datasets. Phys. Med. Biol. 65, 015010 (2020).

24. Orlhac, F. et al. Tumor texture analysis in 18F-FDG PET: relationships between texture parameters, histogram indices, standardized uptake values, metabolic volumes, and total lesion glycolysis. J. Nucl. Med. 55, 414-422 (2014).

25. Hatt, M. et al. Characterization of PET/CT images using texture analysis: the past, the present... any future?. Eur. J. Nucl. Med. Mol. Imaging 44, 151-165 (2017).

26. R Core Team. R: A Language and Environment for Statistical Computing. (2019).

27. Robin, X. et al. pROC: an open-source package for R and S+ to analyze and compare ROC curves. BMC Bioinf. 12, 77 (2011).

28. Wu, Q., Liu, J., Zhang, Y., Wu, S. \& Xie, X. Predictive value of positron emission tomography for the prognosis of immune checkpoint inhibitors in malignant tumors. Cancer Immunol. Immunother. 69, 927-936 (2020).

29. Ahn, H. K., Lee, H., Kim, S. G. \& Hyun, S. H. Pre-treatment 18F-FDG PET-based radiomics predict survival in resected non-small cell lung cancer. Clin. Radiol. 74, 467-473 (2019).

30. Dissaux, G. et al. Pre-treatment 18F-FDG PET/CT radiomics predict local recurrence in patients treated with stereotactic radiotherapy for early-stage non-small cell lung cancer: a multicentric study. J. Nucl. Med. 61, 814-820 (2020).

31. Bundschuh, R. A. et al. Textural parameters of tumor heterogeneity in 18F-FDG PET/CT for therapy response assessment and prognosis in patients with locally advanced rectal cancer. J. Nucl. Med. 55, 891-897 (2014).

32. Brown, P. J. et al. Prediction of outcome in anal squamous cell carcinoma using radiomic feature analysis of pre-treatment FDG PET-CT. Eur. J. Nucl. Med. Mol. Imaging 46, 2790-2799 (2019).

33. Herbst, R. S. et al. Predictive correlates of response to the anti-PD-L1 antibody MPDL3280A in cancer patients. Nature 515, 563-567 (2014).

34. Kim, J. M. \& Chen, D. S. Immune escape to PD-L1/PD-1 blockade: seven steps to success (or failure). Ann. Oncol. 27, 1492-1504 (2016).

35. Hugo, W. et al. Genomic and transcriptomic features of response to Anti-PD-1 therapy in metastatic melanoma. Cell 165, 35-44 (2016).

36. Dittrich, D. et al. Textural features in FGD-PET/CT can predict outcome in melanoma patients to treatment with Vemurafenib and Ipililumab. Nuklearmedizin 59, 228-234 (2020).

37. Galavis, P. E., Hollensen, C., Jallow, N., Paliwal, B. \& Jeraj, R. Variability of textural features in FDG PET images due to different acquisition modes and reconstruction parameters. Acta Oncol. 49, 1012-1016 (2010).

38. Nakajo, M. et al. Application of a machine learning approach for the analysis of clinical and radiomic features of pretreatment [18F]-FDG PET/CT to predict prognosis of patients with endometrial cancer. Mol. Imaging Biol. https://doi.org/10.1007/s11307021-01599-9 (2021).

39. Chen, C. et al. Removing batch effects in analysis of expression microarray data: an evaluation of six batch adjustment methods. PLOS ONE 6, e17238 (2011).

40. Wahl, R. L., Jacene, H., Kasamon, Y. \& Lodge, M. A. From RECIST to PERCIST: evolving considerations for PET response criteria in solid tumors. J. Nucl. Med. 50, 122S-150S (2009).

\section{Acknowledgements}

The authors would like to thank Dr Orlhac for constructive criticism of the manuscript and Dr Simonson for proofreading the article.

\section{Author contributions}

A.F. contributed to data acquisition, to conception and design, to data interpretation and wrote the manuscript. V.H. and N.D.L. contributed to acquire data, revised manuscript and enhanced the intellectual content. F.C. provided expert guidance, contributed to conception and design, revised the manuscript and enhanced the intellectual content. N.P., J.P.V. contributed to revise the manuscript and enhanced the intellectual content. J.L.P. and M.T.L. performed the clinical evaluations, treatments and follow-up evaluations of the patients. All authors reviewed the manuscript.

\section{Competing interests}

The authors declare no competing interests.

\section{Additional information}

Supplementary Information The online version contains supplementary material available at https://doi.org/ 10.1038/s41598-021-98310-3.

Correspondence and requests for materials should be addressed to A.F.

Reprints and permissions information is available at www.nature.com/reprints.

Publisher's note Springer Nature remains neutral with regard to jurisdictional claims in published maps and institutional affiliations. 
(c) (i) Open Access This article is licensed under a Creative Commons Attribution 4.0 International cc) License, which permits use, sharing, adaptation, distribution and reproduction in any medium or format, as long as you give appropriate credit to the original author(s) and the source, provide a link to the Creative Commons licence, and indicate if changes were made. The images or other third party material in this article are included in the article's Creative Commons licence, unless indicated otherwise in a credit line to the material. If material is not included in the article's Creative Commons licence and your intended use is not permitted by statutory regulation or exceeds the permitted use, you will need to obtain permission directly from the copyright holder. To view a copy of this licence, visit http://creativecommons.org/licenses/by/4.0/.

(C) The Author(s) 2021 